\title{
Heat and Mass Transfer of a Chemically Reacting Micropolar Fluid Over a Linear Streaching Sheet in Darcy Forchheimer Porous Medium
}

\author{
S.Rawat \\ Department of Mathematics, \\ Galgotias University, G. Noida \\ S.Kapoor \\ Department of Mathematics, \\ THDC Institute of Hydropower Engineering and \\ Technology, B.Puram, Tehri
}

\author{
R. Bhagrava \\ Department of Mathematics, \\ Indian Institute of Technology, Roorkee \\ O. Anwar. Beg \\ Department of Engineering and Mathematics, Sheaf \\ Building, Sheaf Street, Sheffield Hallam University, \\ Sheffield, S11WB, England.
}

\begin{abstract}
In the present study, an analysis is carried out to study twodimensional, laminar boundary layer flow and mass transfer of a micropolar chemically-reacting fluid past a linearly stretching surface embedded in a porous medium. Such a study finds important applications in geochemical systems and also chemical reactor process engineering. The non-linear partial boundary layer differential equations, governing the problem under consideration, have been transformed by a similarity transformation into a system of ordinary differential equations, which is solved numerically by using the galerkin finite element method. The numerical outcomes thus obtained are depicted graphically to illustrate the effect of different controlling parameters on the dimensionless velocity, temperature and concentration profiles. Comparisons of finite element method and finite difference method is also presented in order to test the accuracy of the methods and the results obtained are found to have an excellent agreement. Finally, the numerical values for quantities of physical interest like local Nusselt number and skin friction are also presented in tabular form.
\end{abstract}

\section{Keyword}

Galerkin Finite Element Method, Skin friction, linearly stretching sheet, chemically reacting fluid.

\section{INTRODUCTION}

Flows with chemical reaction has numerous applications in many branches of engineering science including hypersonic aerodynamics [1,2], geophysics and volcanic systems [3], catalytic technologies [4] and chemical engineering processes [5]. Many such studies have been done with boundary layer theory. Acrivos [6] studied the laminar boundary layer flow with fast chemical reactions. Takhar and Soundalgekar [7] studied the diffusion of a chemically-reacting species in laminar boundary layer flow with suction effects. Later Merkin [8] considered isothermal reactive boundary layer flows. More recently Shateyi et al [9] has studied chemicallyreactive convective boundary layer flows using asymptotic analysis. Little work has been done in analysing the chemically-reactive boundary layer flow in porous media, a topic of great importance in e.g. packed-bed transport processes, geological contamination and also industrial materials processing. Pop et al [10] investigated the effects of both homogenous and heterogeneous chemical reactions on dispersion in porous media using a Darcian formulation. Aharonov et al [11] studied the three-dimensional reactive flow in porous media with dissolution effects. Later Fogler and Fredd [12] analyzed the chemically-reactive flow in porous media. In many industrial processes, engineers are primarily concerned with flow and transport phenomena over accelerating and stretching surfaces. In this regard many studies have also been communicated. Sakiadis [13] first studied the laminar boundary layer flow past a continuous flat surface. Vlegger [14] investigated the boundary layer flow on a continuous accelerating plate. Takhar et al [15] examined the effects of magnetism and chemical reaction on flow and species transfer over a stretching sheet. More recently Acharya et al [16] have modeled the coupled heat and mass transfer with heat source effects on an accelerating surface. Most of these studies were concerned with Newtonian fluids, but in various chemical engineering applications, biomechanics, slurry technologies etc, however,the flow is not newtonian. Keeping all this under consideration, Eringen [17] developed the theory of micropolar fluids seeing the increasing importance of large number of non-Newtonian fluids in processing industries and elsewhere of materials whose flow behavior includes rotating elements at the microscopic level. The theory can be applied successfully to explain the problems of colloidal fluids, liquid crystals, lubricants, suspensions, synovial fluid etc. Eringen [18] later developed the theory of thermomicropolar fluids to include heating effects. Micropolar transport phenomena therefore are important to study from the viewpoint of elucidating more accurately the flow dynamics occurring in many engineering systems. A number of studies in micropolar heat transfer has been communicated in the past three decades. Hassanien and Gorla [19] studied the boundary flow of a micropolar fluid near the stagnation point on a horizontal cylinder. Agarwal et al [20] studied the micropolar heat transfer past a stretching surface. Bhargava et al [21] examined the micropolar flow between rotating discs. Beg .et.al [26] has investigated the heat and mass transfer phenomena in porous media using microplar fluid, and then they used computational finite element technique for a two dimensional problem in channel [27]. In this continuation Rawat.et.al [28], has used the above technique for the heat and mass transfer phenomena while incorporating the soret and duffor effects, hence investigated the theremophysical effects using MHD micropolar fluid in porous media[29]. Recently Usman.et.al, [30-31] has pointed out some aspect while focusing at Unsteady MHD micropolar Flow and Mass Transfer Past a Vertical Permeable Plate with 
Variable Suction and then attempting the problem while incorporating the chemical reaction parameter in RadiationConvection Flow in Porous Medium but still many later studies however did not consider the influence of chemical reaction or species transfer on the flow regime. In the present study, we consider numerically the buoyancy-induced convective flow and mass transfer of a micropolar, chemically-reacting fluid over a vertical stretching plane embedded in a DF porous medium. The FEM has been utilized to solve the mathematical model which constitutes a two-point boundary value problem. Such a study finds important applications in geochemical systems and also chemical reactor process engineering

\section{MATHEMATICAL MODEL}

Consider the two-dimensional, laminar boundary layer flow and mass transfer of a micropolar chemically-reacting fluid past a vertical stretching surface embedded in a porous medium.

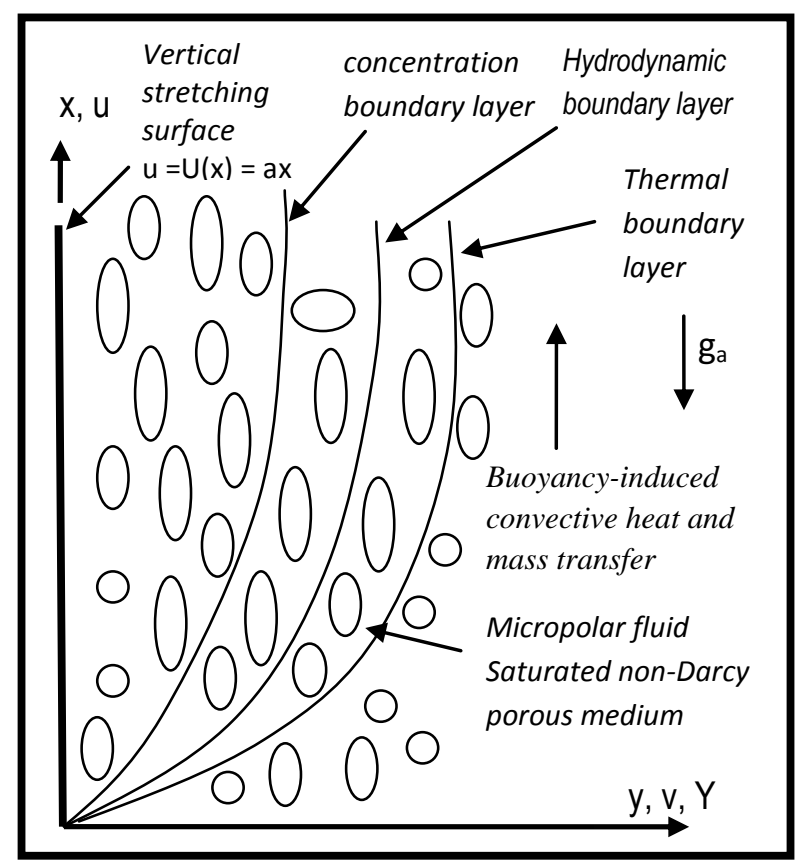

Fig. 1: Physical Model

The $\mathrm{x}$-axis is located parallel to the vertical surface and the $y$ axis perpendicular to it. We assume constant micropolar fluid properties throughout the medium i.e. density, mass diffusivity, viscosity and chemical reaction rate are fixed. Concentration of species in the free stream i.e. far away from the stretching surface, is assumed to be infinitesimal (zero), see [14] and defined as $C_{\infty}$. Temperature in the free stream is taken as $T_{\infty}$. The governing boundary layer equations for the flow regime, illustrated in Fig. 1, incorporating a linear Darcian drag and a second-order Forchheimer drag, takes the following form, under the Boussinesq approximation:

\section{Conservation of Mass:}

$\frac{\partial u}{\partial x}+\frac{\partial v}{\partial y}=0$

$$
\begin{aligned}
u \frac{\partial u}{\partial x}+v \frac{\partial u}{\partial y}= & v_{1} \frac{\partial^{2} u}{\partial y^{2}}+k_{1} \frac{\partial N}{\partial y}+\beta g_{a}\left(h-h_{\infty}\right) \\
& +\beta^{*} g_{a}\left(c-c_{\infty}\right)-\frac{v_{1}}{k_{p}} u-\frac{b}{k_{p}} u^{2}
\end{aligned}
$$

Conservation of Angular Momentum:

$u \frac{\partial N}{\partial x}+v \frac{\partial N}{\partial y}=-\frac{\kappa}{\rho j}\left(2 N+\frac{\partial u}{\partial y}\right)+\frac{\gamma}{\rho j} \frac{\partial^{2} N}{\partial y^{2}}$

Conservation of Energy:

$u \frac{\partial h}{\partial x}+v \frac{\partial h}{\partial y}=\alpha \frac{\partial^{2} h}{\partial y^{2}}$

Conservation of Species:

$u \frac{\partial c}{\partial x}+v \frac{\partial c}{\partial y}=D \frac{\partial^{2} c}{\partial y^{2}}-\Gamma c$.

The corresponding boundary conditions on the vertical surface and in the free stream can be defined now as:

$$
\begin{aligned}
& y=0: u=a x, \quad v=0, \quad h=h_{w}, \quad c=c_{w}, \quad N=-s\left(\frac{\partial u}{\partial y}\right) \\
& y \rightarrow \infty \quad: \quad u=0, \quad h=h_{\infty}, \quad c=c_{\infty}, \quad N \rightarrow 0
\end{aligned}
$$

where $v_{1}=(\mu+\kappa) / \rho$ is the apparent kinematic viscosity and $k_{1}=\kappa / \rho\left(k_{1}>0\right)$ is the coupling constant. Following Crane [22], the surface velocity of the stretching plane is assumed to vary linearly with distance $\mathrm{x}(\mathrm{u}=\mathrm{U}(\mathrm{x})=\mathrm{ax}$, for a $>0$ where a denotes a dimensional constant), $\Gamma$ is the chemical reaction rate parameter. Non-dimensionalizing the conservation equations by introducing the following transformations:

$\psi=\left[v_{1} x U(x)\right]^{1 / 2} f(Y), \quad Y=\left[\frac{U(x)}{v_{1} x}\right]^{1 / 2} y$,

$u=\frac{\partial \psi}{\partial y}, \quad v=-\frac{\partial \psi}{\partial x}, \quad N=\sqrt{\frac{U(x)}{v_{1} x}} U(x) g(Y)$,

$\mathrm{U}(\mathrm{x})=\mathrm{ax}, \quad \theta=\frac{h-h_{\infty}}{h_{w}-h_{\infty}}, \quad C=\frac{c-c_{\infty}}{c_{w}-c_{\infty}}$,

Equation (5.8) reduces the above set of equations (5.1)-(5.5) into the following set of ordinary differential equations:

\section{Conservation of Momentum:}

$$
\begin{aligned}
& \frac{d^{3} f}{d Y^{3}}+B_{1} \frac{d g}{d Y}+f \frac{d^{2} f}{d Y^{2}}-\left(\frac{d f}{d Y}\right)^{2}+G r_{x} \operatorname{Re}_{x} \theta \\
& +G c_{x} \operatorname{Re}_{x} C-\frac{1}{\operatorname{Re}_{x} D a_{x}} \frac{d f}{d Y}-\frac{F n_{x}}{D a_{x}}\left(\frac{d f}{d Y}\right)^{2}=0
\end{aligned}
$$

\section{Conservation of Momentum:}


Conservation of Angular Momentum:

$\lambda \frac{d^{2} g}{d Y^{2}}-\frac{\lambda}{\Lambda}\left(2 g+\frac{d^{2} f}{d Y^{2}}\right)-\frac{d f}{d Y} g+f \frac{d g}{d Y}=0$

Conservation of Energy:

$\frac{d^{2} \theta}{d Y^{2}}+\operatorname{Pr} f \frac{d \theta}{d Y}=0$

\section{Conservation of Species:}

$\frac{d^{2} C}{d Y^{2}}+S c f \frac{d C}{d Y}-S c\left[\chi \operatorname{Re}_{x} C+C \frac{d f}{d Y}\right]=0$,

where:

$$
\begin{aligned}
& G c_{x}=\frac{v_{1} g_{a} \beta *\left[c_{w}-c_{\infty}\right]}{U^{3}}, G r_{x}=\frac{v_{1} g_{a} \beta\left[h_{w}-h_{\infty}\right]}{U^{3}}, \\
& D a_{x}=\frac{k_{p}}{x^{2}}, \quad F n_{x}=\frac{b}{x}, \quad B=\frac{k_{1} \rho}{\mu}=\frac{\kappa}{\mu}, \\
& \operatorname{Re}_{x}=\frac{U x}{v_{1}}, \quad \operatorname{Pr}=\frac{v_{1}}{\alpha}, \quad S c=\frac{v_{1}}{D}, \quad \chi=\frac{v_{1} \Gamma}{U^{2}} .
\end{aligned}
$$

The corresponding boundary conditions (5.6)-(5.7) are transformed as follows:

$$
\begin{gathered}
\text { At } Y=0: \quad f(0)=0 ; \quad \frac{d f}{d Y}(0)=1 ; \quad \theta(0)=1 \text { and } \\
C(0)=1 ; \quad g(0)=-s \frac{d^{2} f}{d Y^{2}}(0)
\end{gathered}
$$

And as

$$
Y \rightarrow \infty: \frac{d f}{d Y} \rightarrow 0 ; \quad \theta \rightarrow 0 ; \quad C \rightarrow 0 ; \quad g \rightarrow 0 .
$$

The shear stress on the sheet surface at $s=0.5$ is defined as:

$$
\begin{aligned}
& \tau_{w}=\left[(\mu+\kappa) \frac{d u}{d y}+\kappa N\right]_{y=0} \\
& =\left((\mu+\kappa) U\left(\frac{U}{v_{1} x}\right)^{1 / 2} f^{\prime \prime}(0)-\frac{\kappa}{2} U\left(\frac{U}{v_{1} x}\right)^{1 / 2} f^{\prime \prime}(0)\right)
\end{aligned}
$$

whereas the skin friction coefficient is defined by:

$$
C_{f}=\frac{\tau_{w}}{\rho u^{2}} \quad \Rightarrow \quad C_{f}\left(\operatorname{Re}_{x}\right)^{1 / 2}=\left(1+\frac{B_{1}}{2}\right) f^{\prime \prime}(0) .
$$

The heat flux at the sheet surface may be written using Fourier's law as follows:

$$
q_{w}=-\left.k \frac{\partial h}{\partial y}\right|_{y=0}=-k\left(h_{w}-h_{\infty}\right)\left(\frac{U}{v_{1} x}\right)^{1 / 2} \theta^{\prime}(0),
$$

where $k$ is the coefficient of thermal conductivity. The heat transfer coefficient is given by:

$h_{f}=\frac{q_{w}}{\left(h_{w}-h_{\infty}\right)}=-k\left(\frac{U}{v_{1} x}\right)^{1 / 2} \theta^{\prime}(0)$.

The Local Nusselt number can be written as:

$$
N u_{x}=\frac{h_{f} x}{k}=-\left(\operatorname{Re}_{x}\right)^{1 / 2} \theta^{\prime}(0)
$$

\section{NUMERICAL SOLUTION}

Finite element solution to the governing flow equations (5.9) to (5.12) with corresponding boundary conditions (5.14) and (5.15) has been obtained. Assuming that

$$
\frac{d f}{d Y}=U
$$

the equations (5.9) to (5.12) are therefore reduced to the following, where '(dash) indicates d/dY:

$$
\begin{aligned}
& \lambda g^{\prime \prime}-\frac{\lambda}{\Lambda}\left(2 g+U^{\prime}\right)-U g+f g^{\prime}=0 \\
& \theta^{\prime \prime}+\operatorname{Pr} f \theta^{\prime}=0 \\
& C^{\prime \prime}+S c f C^{\prime}-S c \operatorname{Re}_{x} \chi C=0,
\end{aligned}
$$$$
U^{\prime \prime}+B_{1} g^{\prime}+f U^{\prime}+G r \operatorname{Re}_{x} \theta+G c_{x} \operatorname{Re}_{x} C-U^{2}
$$$$
-\frac{1}{D a_{x} \operatorname{Re}_{x}} U-\frac{F n_{x}}{D a_{x}} U^{2}=0
$$

with the corresponding boundary conditions:

$$
\begin{aligned}
& \text { At } \mathrm{Y}=0: \quad \mathrm{f}(0)=0, \quad \mathrm{U}(0)=1 \text { and } \\
& \quad \theta(0)=1, \quad C(0)=1, \quad g(0)=-s U^{\prime}(0)
\end{aligned}
$$

For computational purposes and without loss of generality, $\infty$ has been fixed as 8 , with numerical justification. The whole domain is divided into a set of 80 line elements of equal width, each element being two-noded.

\subsection{Variation formulation}

The variational form associated with equations (21)-(25) over a typical two noded-linear element is given by

$\int_{Y_{e}}^{Y_{e+1}} w_{1}\left\{f^{\prime}-U\right\} d Y=0$ 
$\int_{Y_{e}}^{Y_{e+1}} w_{2}\left\{\begin{array}{l}U^{\prime \prime}+B_{1} g^{\prime}+f U^{\prime}+G r_{x} \operatorname{Re}_{x} \theta \\ +G c_{x} \operatorname{Re}_{x} C-U^{2}-\frac{1}{D a_{x} \operatorname{Re}_{x}} U-\frac{F n_{x}}{D a_{x}} U^{2}\end{array}\right\} d Y=0$

$\int_{Y_{e}}^{Y_{e+1}} w_{3}\left\{\lambda g^{\prime \prime}-\frac{\lambda}{\Lambda}\left(2 g+U^{\prime}\right)-U g+f g^{\prime}\right\} d Y=0$

$Y_{e+1}$

$\int_{Y_{e}}^{Y_{e+1}} w_{4}\left\{\theta^{\prime \prime}+\operatorname{Pr} f \theta^{\prime}\right\} d Y=0$

$Y_{e}$

$\int_{Y_{e}}^{Y_{e+1}} w_{5}\left\{C^{\prime \prime}+S c f C^{\prime}-S c \operatorname{Re}_{x} \chi C\right\} d Y=0$,

where $w_{1}, w_{2}, w_{3}, w_{4}$ and $w_{5}$ are arbitrary test functions and may be viewed as the variation in $f, U, g, \theta$ and $\mathrm{C}$ respectively.

\subsection{Finite element formulation}

The finite element model may be obtained from equations (28)-(32) by substituting finite element approximations of the form:

$$
\begin{gathered}
f=\sum_{j=1}^{2} f_{j} \psi_{j}, \quad U=\sum_{j=1}^{2} U_{j} \psi_{j}, \quad g=\sum_{j=1}^{2} g_{j} \psi_{j}, \\
\theta=\sum_{j=1}^{2} \theta_{j} \psi_{j}, \quad C=\sum_{j=1}^{2} C_{j} \psi_{j},
\end{gathered}
$$$$
\text { with } w_{1}=w_{2}=w_{3}=w_{4}=w_{5}=\psi_{i} \quad(i=1,2),
$$

Here $\psi_{i}$ are the shape functions for a typical element $\left(Y_{e}, Y_{e+1}\right)$ and are taken as:

$$
\psi_{1}^{(e)}=\frac{Y_{e+1}-Y}{Y_{e+1}-Y_{e}}, \quad \psi_{2}^{(e)}=\frac{Y-Y_{e}}{Y_{e+1}-Y_{e}}, \quad Y_{e} \leq Y \leq Y_{e+1} .
$$

Using equations (33) - (35), equations (28) to (32) become as:

$$
\int_{Y_{e}}^{Y_{e+1}}\left\{\psi_{i} \sum_{j=1}^{2} f_{j} \frac{d \psi_{j}}{d Y}-\psi_{i} \sum_{j=1}^{2} U_{j} \psi_{j}\right\} d Y=0
$$

$\int_{Y_{e}}^{Y_{e+1}}\left\{\begin{array}{c}-\frac{d \psi_{i}}{d Y} \sum_{j=1}^{2} U_{j} \frac{d \psi_{j}}{d Y}+B_{1} \psi_{i} \sum_{j=1}^{2} g_{j} \frac{d \psi_{j}}{d Y}+ \\ \psi_{i} \frac{f}{d}\left(\sum_{j=1}^{2} U_{j} \frac{d \psi_{j}}{d Y}\right)+G r_{x} \operatorname{Re}_{x} \psi_{i} \sum_{j=1}^{2} \theta_{j} \psi_{j}\end{array}\right\} d Y$

$$
+\int_{Y_{e}}^{Y_{e+1}}\left\{\begin{array}{l}
G c_{x} \operatorname{Re}_{x} \psi_{i} \sum_{j=1}^{2} C_{j} \psi_{j}-\psi_{i} \bar{U}\left(\sum_{j=1}^{2} U_{j} \psi_{j}\right) \\
-\frac{1}{D a_{x} \operatorname{Re}_{x}} \psi_{i}\left(\sum_{j=1}^{2} U_{j} \psi_{j}\right)-\frac{F n_{x}}{D a_{x}} \psi_{i} \bar{U}\left(\sum_{j=1}^{2} U_{j} \psi_{j}\right)
\end{array}\right\} d Y
$$

$$
=-\left(\psi \frac{d U}{i} \frac{Y}{d Y}\right)_{Y}^{e+1}
$$

$$
\begin{gathered}
\int_{e+1}^{Y_{e}}\left\{\begin{array}{c}
-\lambda \frac{d \psi_{i}}{d Y} \sum_{j=1}^{2} g_{j} \frac{d \psi_{j}}{d Y}-\frac{\lambda}{\Lambda} \psi_{i}\left(2 \sum_{j=1}^{2} g_{j} \psi_{j}+\sum_{j=1}^{2} U_{j} \frac{d \psi_{j}}{d Y}\right) \\
-\psi_{i} \bar{U}\left(\sum_{j=1}^{2} g_{j} \psi_{j}\right)+\psi_{i} \bar{f}\left(\sum_{j=1}^{2} g_{j} \frac{d \psi_{j}}{d Y}\right)^{d}
\end{array}\right\} d Y \\
=-\lambda\left(\psi \frac{d g}{i}\right)_{Y}^{Y} e+1
\end{gathered}
$$

$\int_{Y_{e}}^{Y_{e+1}}\left\{-\frac{d \psi_{i}}{d Y} \sum_{j=1}^{2} \theta_{j} \frac{d \psi_{j}}{d Y}+\operatorname{Pr} \psi_{i} \bar{f}\left(\sum_{j=1}^{2} \theta_{j} \frac{d \psi_{j}}{d Y}\right)\right\} d Y$

$$
=-\left(\psi \frac{d \theta}{d Y}\right)_{Y}^{Y} e+1
$$

$$
\int_{Y_{e}}^{Y_{e+1}}\left\{\begin{array}{l}
-\frac{d \psi_{i}}{d Y} \sum_{j=1}^{2} C_{j} \frac{d \psi_{j}}{d Y}+S c \psi_{i} \bar{f}\left(\sum_{j=1}^{2} C_{j} \frac{d \psi_{j}}{d Y}\right) \\
-S c \operatorname{Re}_{x} \chi \psi_{i}\left(\sum_{j=1}^{2} C_{j} \psi_{j}\right)
\end{array}\right\} d Y
$$

$$
=-\left(\psi \frac{d C}{i} \frac{Y}{d Y}\right)_{Y_{e}}^{e+1} .
$$

The finite element model of the equations thus formed is given by:

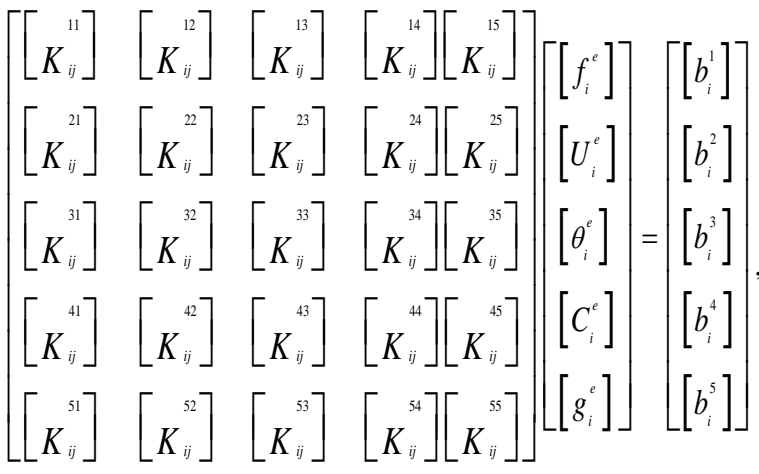

where $\left[K_{i j}^{m n}\right]$ and $\left[b_{i}^{m}\right](m, n=1,2,3,4,5$ and $i, j=1,2)$ are the matrices of order $2 \times 2$ and $2 \times 1$ respectively. Also $\left[f_{i}^{e}\right],\left[U_{i}^{e}\right],\left[\theta_{i}^{e}\right],\left[C_{i}^{e}\right]$ and $\left[g_{i}^{e}\right]$ are matrices of order $2 \times 1$. All these matrices may be defined as follows: 


$$
\begin{aligned}
& K_{i j}^{11}=\int_{Y_{e}}^{Y_{e+1}} \psi_{i} \frac{d \psi}{d Y} d Y, K_{i j}^{12}=-\int_{Y_{e}}^{Y_{e+1}} \psi_{i} \psi_{j} d Y, \\
& K_{i j}^{13}=K_{i j}^{14}=K_{i j}^{15}=0 \\
& K_{i j}^{21}=0 \text {, } \\
& K_{i j}^{22}=-\int_{\substack{Y \\
e}}^{Y} \frac{d \psi_{i}}{d Y} \frac{d \psi}{d Y} d Y+\bar{f}_{1} \int_{Y_{e}}^{Y+1} \psi_{i} \psi_{1} \frac{d \psi}{d Y} d Y \\
& +\bar{f}_{2} \int_{Y_{e}}^{Y^{++1}} \psi_{i} \psi_{2} \frac{d \psi}{d Y} d Y-\bar{U}_{1} \int_{Y_{e}}^{Y_{e+1}} \psi_{i} \psi_{1} \psi_{j} d \eta \\
& -\bar{U}_{2} \int_{Y_{e}}^{Y_{e+1}} \psi_{i} \psi_{2} \psi_{j} d Y-\frac{1}{\operatorname{Da}_{x} \operatorname{Re}_{x}} \int_{Y_{e}}^{Y_{e+1}} \psi_{i} \psi_{j} d Y \\
& -\frac{F n_{x}}{D a_{x}} \bar{U}_{1} \int_{Y_{e}}^{Y_{e+1}} \psi_{i} \psi_{1} \psi_{j} d \eta-\frac{F n_{x}}{D a_{x}} \bar{U}_{2} \int_{Y_{e}}^{Y_{e+1}} \psi_{i} \psi_{2} \psi_{j} d Y=0 \text {, } \\
& \int_{Y_{e}}^{Y_{e+1}} \psi_{i} \psi_{j} d Y, \quad K_{i j}^{24}=G c_{x} \operatorname{Re}_{x} \int_{Y_{e}}^{Y_{e+1}} \psi_{i} \psi{ }_{j} d Y, \\
& K_{i j}^{25}=B_{1} \int_{Y_{e}}^{Y_{e}+1} \psi_{i} \frac{d \psi}{d Y} d Y, \\
& K_{i j}^{31}=0, \quad K_{i j}^{32}=-\frac{\lambda}{\Lambda} \int_{\substack{Y \\
e}}^{Y_{e+1}} \psi_{i} \frac{d \psi}{d Y} d Y, \quad K_{i j}^{33}=K_{i j}^{34}=0 \\
& K_{i j}^{35}=-\lambda \int_{Y_{e}}^{Y_{e+1}} \frac{d \psi}{d Y} \frac{d \psi}{d Y} d Y-\frac{2 \lambda}{\Lambda} \int_{Y_{e}}^{Y_{e+1}} \psi_{i} \psi_{j} d \eta \\
& -\bar{U}_{1} \int_{Y_{e}}^{Y_{e}} \psi_{i} \psi_{1} \psi_{j} d Y-\bar{U}_{2} \int_{Y_{e}}^{Y^{++1}} \psi_{i} \psi_{2} \psi_{j} d Y \\
& +\bar{f}_{1}^{Y_{e+1}} \int_{Y_{e}} \psi_{i} \frac{d \psi}{d Y} d Y+\bar{f}_{2} \int_{Y_{e}}^{Y_{e+1}} \psi_{i} \psi{ }_{2} \frac{d \psi}{d Y} d Y
\end{aligned}
$$

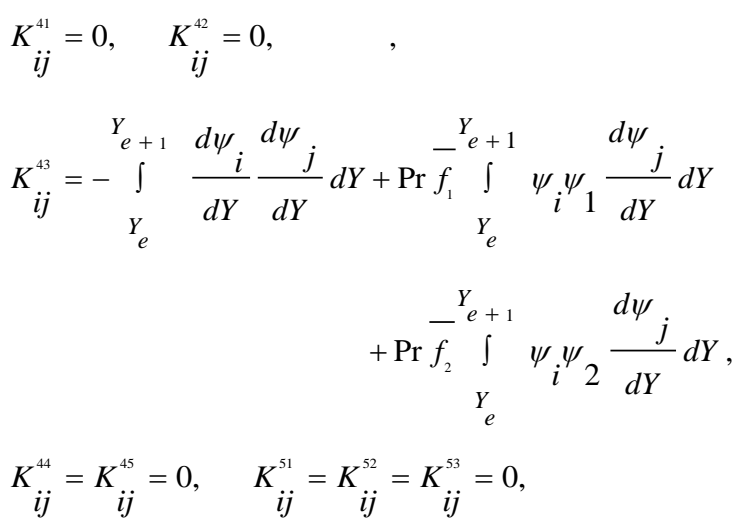$$
K_{i j}^{54}=-\int_{Y_{e}}^{Y_{e+1}} \frac{d \psi}{d Y} \frac{d \psi}{d Y} d Y+S c f_{1}^{Y_{e+1}} \psi_{Y_{e}} \psi_{i} \psi_{1} \frac{d \psi}{d Y} d Y
$$$$
+S c \bar{f}_{2} \int_{Y_{e}}^{Y_{e+1}} \psi_{i} \psi_{2} \frac{d \psi}{d Y} d Y-S c \operatorname{Re}_{x} \chi \int_{Y_{e}}^{Y_{e+1}} \psi_{i} \psi_{j} d Y,
$$$$
K_{i j}^{55}=0
$$$$
b_{i}^{1}=0, b_{i}^{2}=-\left(\psi_{i} \frac{d U}{d Y}\right)_{Y_{e}}^{Y_{e+1}}, \quad b_{i}^{3}=-\lambda\left(\psi_{i} \frac{d g}{d Y}\right)_{Y_{e}}^{Y_{e}+1},
$$$$
b_{i}^{4}=-\left(\psi_{i} \frac{d \theta}{d Y}\right)_{Y_{e}}^{Y_{e}+1}, \quad b_{i}^{5}=-\left(\psi_{i} \frac{d C}{d Y}\right)_{Y_{e}}^{Y_{e+1}},
$$

where

$$
\bar{f}=\sum_{i=1}^{2} \bar{f}_{i} \psi_{i}, \quad \bar{U}=\sum_{i=1}^{2} \overline{U_{i}} \psi_{i} .
$$

Each element matrix given by equation (5.41) is of the order $10 \times 10$. Here, we divide the whole domain into 80 equal line elements. A matrix of order $405 \times 405$ is attained on assembly of all the element equations. The nonlinear system obtained after assembly is linearized by incorporating the functions $\bar{f}$ and $\bar{U}$, which are assumed to be known. Here $\bar{f}_{i}$ and $\bar{U}_{i}$ are the value of the functions $\bar{f}$ and $\bar{U}$ at the $\mathrm{i}^{\text {th }}$ node. A system of 346 equation left after applying the given boundary conditions is solved using an iterative scheme maintaining an accuracy of 0.0005 .

\section{RESULTS AND DISCUSSION}

The following parameter values are adopted in the computations, viz, $G r_{x}=1.0, G c_{x}=1.0, \chi=1.0, D a_{x}=1.0$, $F n_{x}=1.0, R_{x}=1.0, \operatorname{Pr}=0.7, S c=0.1, B_{1}=0.01, \Lambda=1, \lambda=$ 1 and $s=0.5$. The results are computed to see the effect of selected important parameters namely $G r_{x}, G c_{x}, \chi, D a_{x}, F n_{x}$, $\operatorname{Re}_{x}, \operatorname{Pr}, S c, B_{1}, \Lambda, \lambda$ and $s$. 
In Fig. 2, the variation of velocity versus Y, for various values of the chemical reaction number $(\chi)$ are shown. A rise in $\chi$ generates a substantial decrease in velocities. For all values of $\chi$ the profiles descend from unity at the wall $(\mathrm{Y}=0)$, and tend asymptotically to zero at the freestream $(\mathrm{Y} \rightarrow \infty)$. Therefore clearly chemical reaction induces a deceleration in the flow field. In equation (5.12) we observe that the chemical reaction term is negative and indeed opposite to the principal diffusion terms. Therefore logically, chemical reaction will delay diffusive transport which in turn will correspond to retardation in the flow field. Therefore maximum velocity values correspond to the case of zero chemical reaction i.e. $\chi=0$.

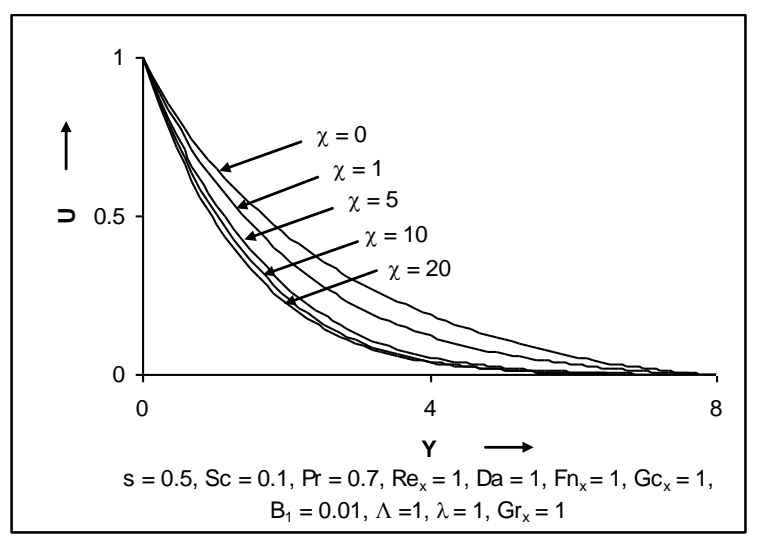

Fig. 2: Velocity distribution for different $\chi$

Conversely, we observe that temperature function profiles i.e. $\theta$ increase with a rise in chemical reaction parameter, as depicted in Fig. 3. The profiles are not as widely dispersed as for the velocity distributions; however there is a clear boost in temperatures especially at intermediate separation from the wall. Our results agree quite well for both velocity and temperature distributions with those due to Afify [23] who considered chemical reaction effects on free convective flow and mass transfer of a viscous, incompressible and electrically conducting fluid over a stretching surface in the presence of a constant transverse magnetic field. Temperature profiles generally are lower in case with no chemical reaction.

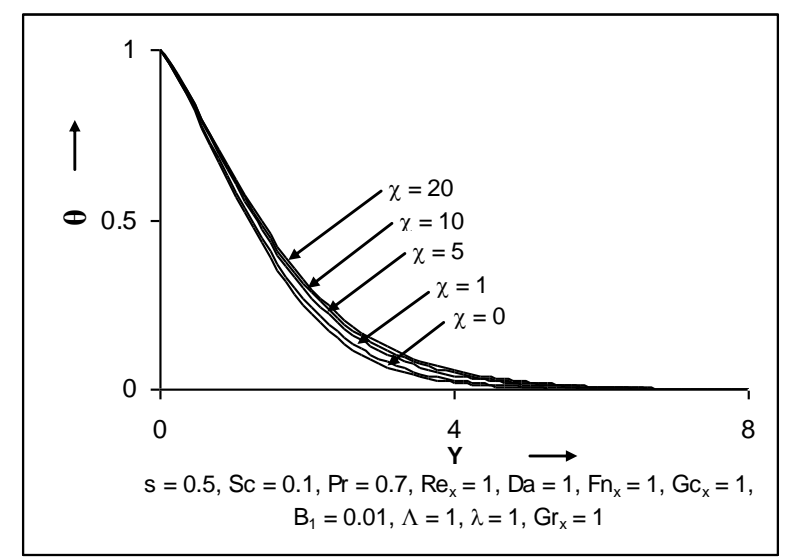

Fig. 3: Temperature distribution for different $\chi$

The influence of $\chi$ on the mass transfer function (C) is plotted in Fig. 4. As $\chi$ increases, concentration decreases. For the non-reactive case, $\chi=0$, there is approximately a linear decay in $\mathrm{C}$ from a maximum at the wall to zero at the free stream, these end values being a direct result of the imposed boundary conditions. As $\chi$ increases the profiles become more monotonic in nature; in particular the gradient of the profile becomes much steeper for $\chi=5$ than for lower values of the chemical reaction parameter. This steepness in the behaviour of $\mathrm{C}$ increases in the vicinity of the stretching surface for $\chi=20$. Chemical reaction parameter therefore has a considerable influence on both magnitude and rate of change of species (mass) transfer function at higher values, since physically this corresponds to faster rate of reaction.

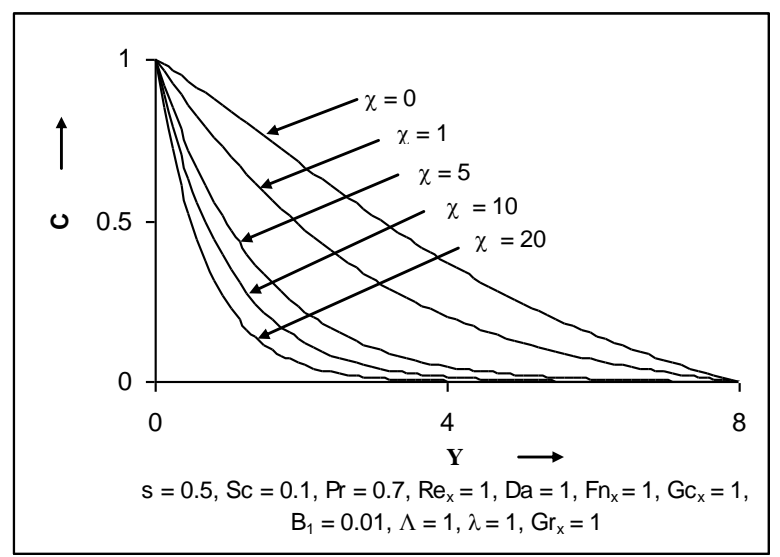

Fig. 4: Concentration distribution for different $\chi$

The response of the micro-rotation profile, illustrated in Fig. 5, to increasing chemical reaction rate parameter is also interesting. We observe that near the wall, micro-rotation increases with a rise in reaction parameter; however away from the it, all profiles converge and a switch over in behaviour occurs, so that micro-rotation is actually depressed by increasing chemical reaction parameter for the rest of the domain, finally converging to zero.

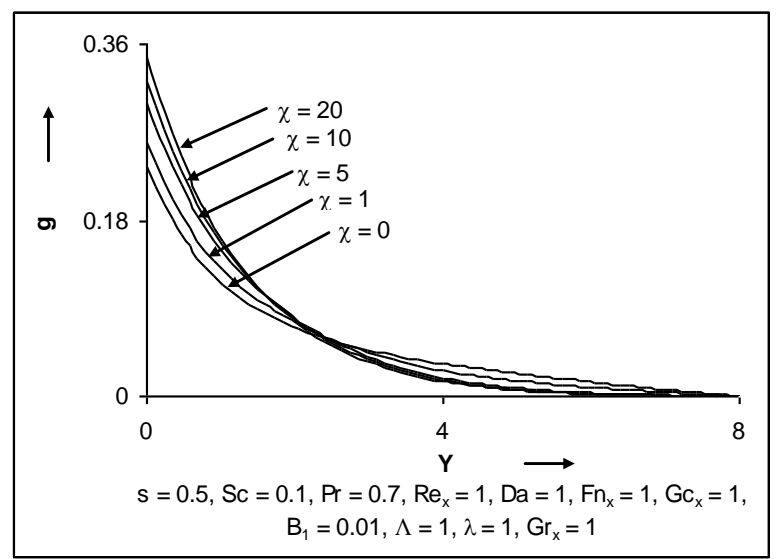

Fig. 5: Microrotation distribution for different $\chi$

The effect of $\mathrm{Gr}_{\mathrm{x}}$ and $\mathrm{Gc}_{\mathrm{x}}$ are shown in Figs. 6 to 8 and Figs 9 to 10 respectively. Fig. 6 contains variation of velocity $\mathrm{U}$ for various $\mathrm{Gr}_{\mathrm{x}}$. This parameter $\left(\mathrm{Gr}_{\mathrm{x}}\right)$ embodies the ratio of the thermal buoyancy force to the viscous hydrodynamic force and therefore is expected to accelerate the flow, a trend confirmed by our results. It is observed that a rise in $\mathrm{Gr}_{\mathrm{x}}$ corresponds to an increase in velocity. This boost is particularly pronounced near the wall, where there is a sharp rise from the stretching surface (wall) especially for the cases $\mathrm{Gr}_{\mathrm{x}}=5$ and 10. Peak velocity for $\mathrm{Gr}_{\mathrm{x}}$ is about 1.5. All profiles generally descend smoothly towards zero although the rate of descent is greater corresponding to higher Grashof numbers. 
Schmidt number has been fixed at 0.1 which physically corresponds to for e.g. Carbon Dioxide gas diffusing through air [24] for which Pr is 0.7 .

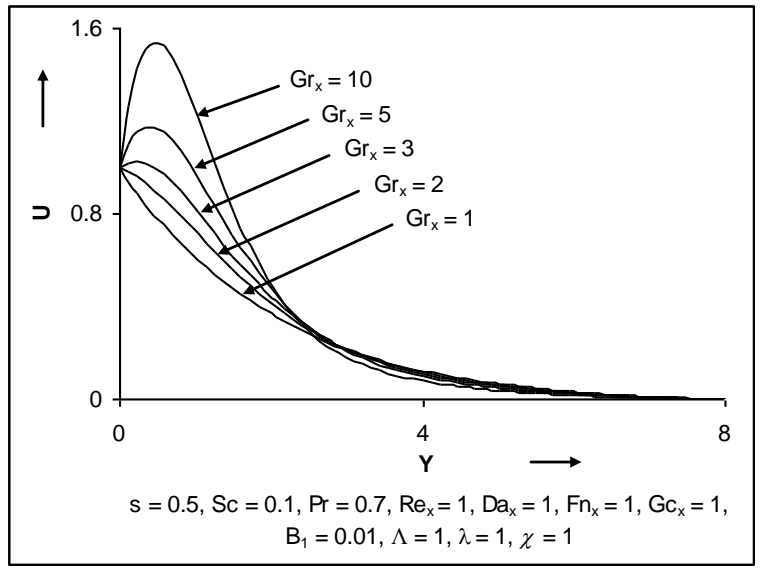

Fig. 6: Velocity distribution for different $\mathbf{G r}_{\mathrm{x}}$

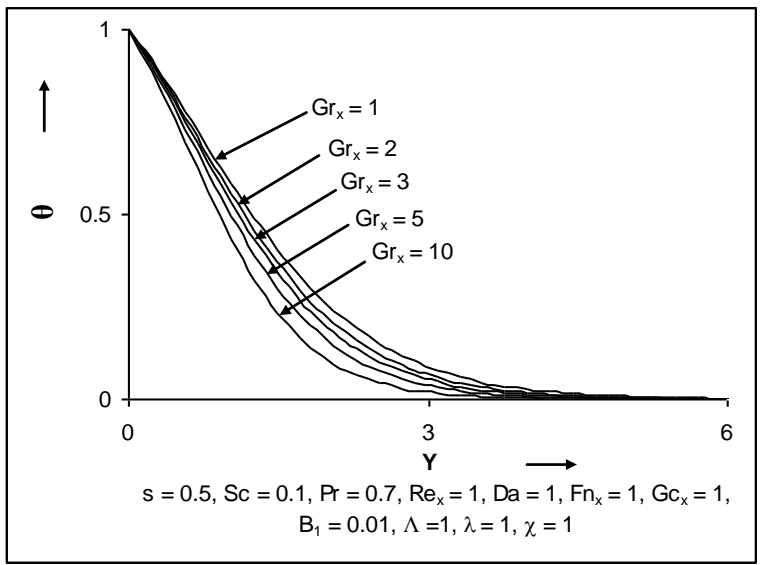

Fig. 7 Temperature distribution for different $\mathbf{G r}_{\mathbf{x}}$

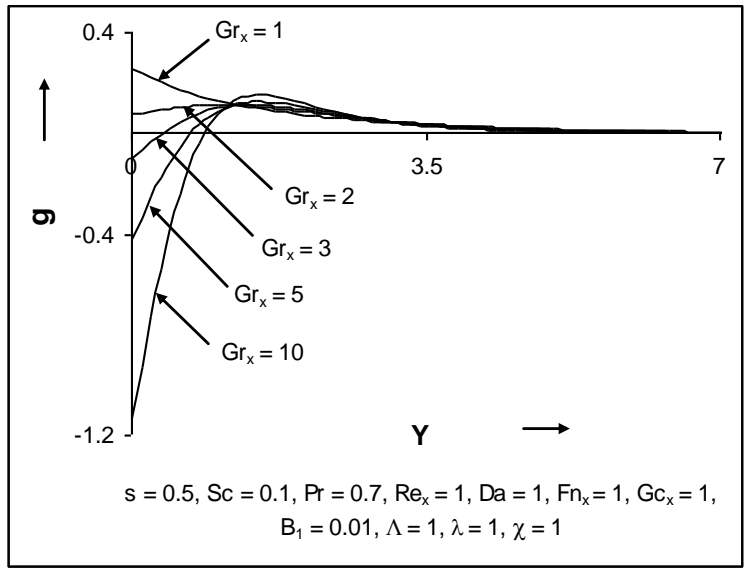

Fig. 8 Microrotation distribution for different $\mathbf{G r}_{\mathbf{x}}$

Temperature distribution $\theta$ versus $\mathrm{Y}$ is plotted in Fig 7 for various $\mathrm{Gr}_{\mathrm{x}}$. It is observed that an increase in $\mathrm{Gr}_{\mathrm{x}}$ decreases temperature in the micropolar fluid. This fall is most apparent between $\mathrm{Y}=1$ to 3 ; all temperatures fall asymptotically to zero as $\mathrm{Y} \rightarrow \infty$.
The micro-rotation profiles also decreases as $\mathrm{Gr}_{\mathrm{x}}$ increases (Fig. 8); in fact they switch from positive values for $\mathrm{Gr}_{\mathrm{x}}=1,2$ to negative values for $\mathrm{Gr}_{\mathrm{x}}=3,5,10$. Near to the wall, all values converge and then descend smoothly to zero. The positive values of micro-rotation indicate spin in one direction and negative values indicate a reverse spin. Buoyancy effects strongly influences the spin of microelements in the micropolar fluid, a feature which is important in various chemical reactor designs.

In Figs. 9 to 10 we have presented the effect of the species Grashof number, $\mathrm{Gc}_{\mathrm{x}}$, on the velocity and concentration profile (in the presence and absence of chemical reaction) respectively. As expected, a distinct increase in velocity $\mathrm{U}$ i.e. $f^{\prime}$, is observed as $\mathrm{Gc}_{\mathrm{x}}$ increases. The general trends for the reactive and non-reactive case appear to be similar; however Fig. 9 clearly shows that the profiles of velocity $(\mathrm{U})$ for the non-reactive case $(\chi=0)$ are greater in value across the domain compared with the reactive regime case $(\chi=1)$.

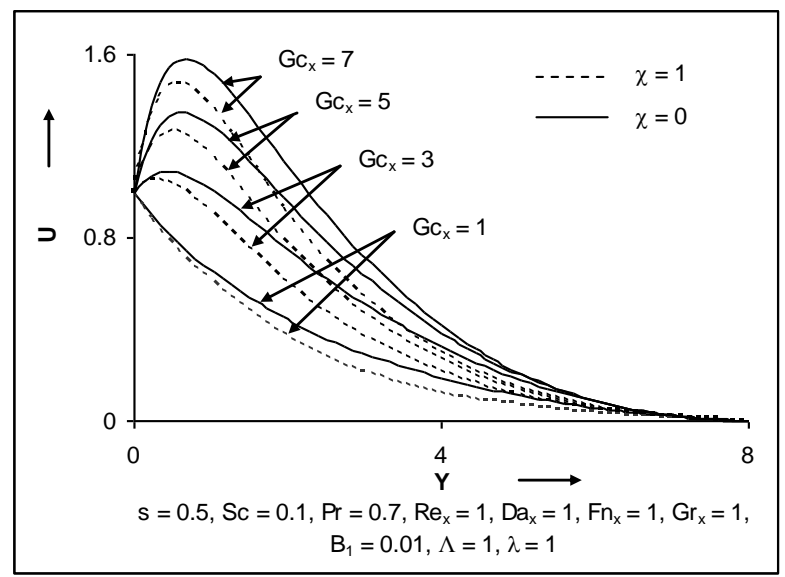

Fig. 9: Velocity distribution for different $\mathbf{G c}_{\mathrm{x}}$

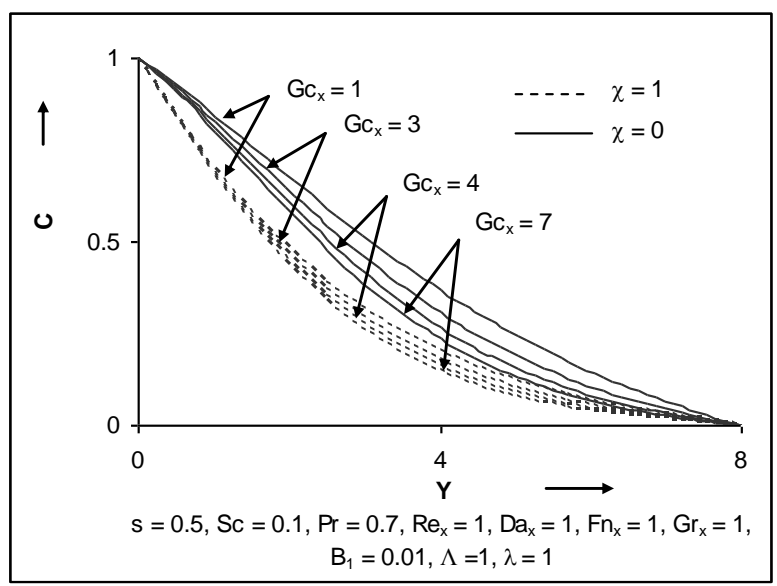

Fig. 10 Concentration distribution for different $\mathbf{G c}_{\mathrm{x}}$

Also species transfer function $\mathrm{C}$, is also affected by increasing $\mathrm{Gc}_{\mathrm{x}}$ (Fig. 10). A rise in $\mathrm{Gc}_{\mathrm{x}}$ corresponds to an decrease in concentration profile. Increasing $\mathrm{Gc}_{\mathrm{x}}$ therefore serves to lower the mass transfer functions throughout the flow field. Such trends are important in environmental flows and also industrial transport phenomena indicating that even in micropolar fluids, increasing buoyancy only boosts the translational velocity but reduces species function. Also the 
concentration profile is greater for the non-reactive case ( $\chi=0)$ as compared to the non-reactive case $(\chi=1)$ with the variation in $\mathrm{Gc}_{\mathrm{x}}$. Chemical reaction therefore clearly serves to decelerate the velocity as well as concentration profiles, as indicated in the earlier Figs. ( 2 and 4.)

The influence of the bulk matrix parameter, $\mathrm{Da}_{\mathrm{x}}$, on the flow field is depicted in Figs. 11 to 13. From Fig. 11 it is clear that a rise in $\mathrm{Da}_{\mathrm{x}}$ i.e rise in permeability increases considerably the translational velocity. With increasing permeability the porous matrix structure becomes less and less prominent and in the limiting case when $D a_{x} \rightarrow \infty$ values, the porosity disappears. The Darcian body force is inversely proportional to $\mathrm{Da}_{\mathrm{x}}$ i.e. larger $\mathrm{Da}_{\mathrm{x}}$ generate lower porous bulk retarding forces. The presence of a porous medium with low permeability therefore can be used as a mechanism for depressing velocities i.e. decelerating flow in industrial applications.

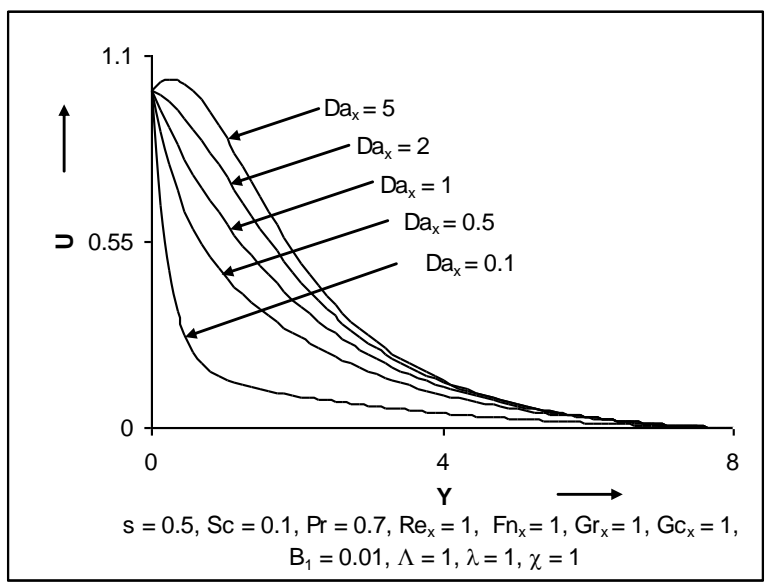

Fig. 11 Velocity distribution for different $\mathrm{Da}_{\mathrm{x}}$

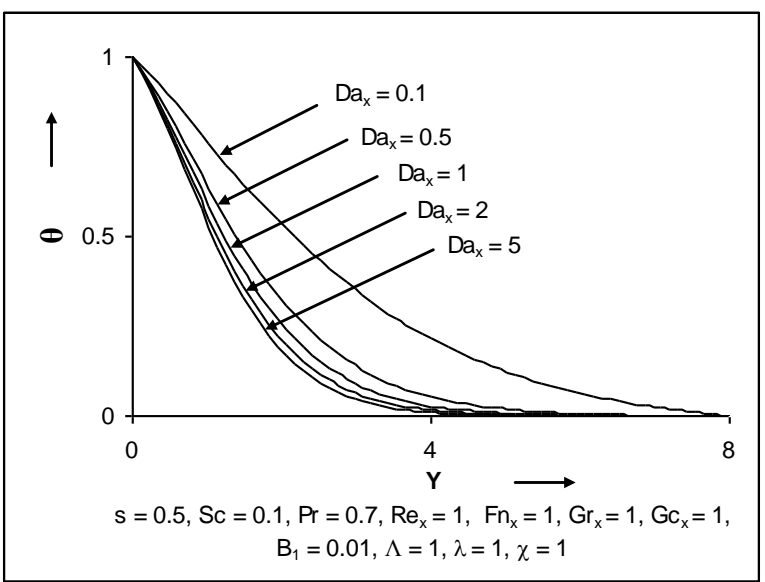

Fig 12 Temperature distribution for different $\mathrm{Da}_{\mathrm{x}}$

Conversely we observe that temperature profiles decreases (Fig. 12) with a rise in $\mathrm{Da}_{\mathrm{x}}$, indicating that progressively less solid matrix particles decrease temperatures in the domain. Conduction heat transfer clearly decreases as solid material vanishes and therefore temperatures for less permeable media $\left(\mathrm{Da}_{\mathrm{x}}=0.1\right)$ are higher than for more permeable media $\left(\mathrm{Da}_{\mathrm{x}}=5\right)$

Increasing Darcy number near the wall serves to lower the micro-rotation of the micropolar fluid, as depicted in Fig. 13. Values of $\mathrm{g}$ at the wall $(\mathrm{Y}=0)$, are initially decreased as $\mathrm{Da}_{\mathrm{x}}$ rises; however away from the wall, an increase in Darcy number serves to enhance the microrotation values. We may infer that close to the wall, microrotation is inhibited even for more permeable media as the particles have difficulty in rotating due to the presence of the wall; however away from the wall, with a more permeable environment, the micropolar spin is not inhibited and microelements can rotate more freely, as demonstrated by the slightly larger values of $\mathrm{g}$ for $\mathrm{Da}_{\mathrm{x}}=5$ at some distance away from the plate.

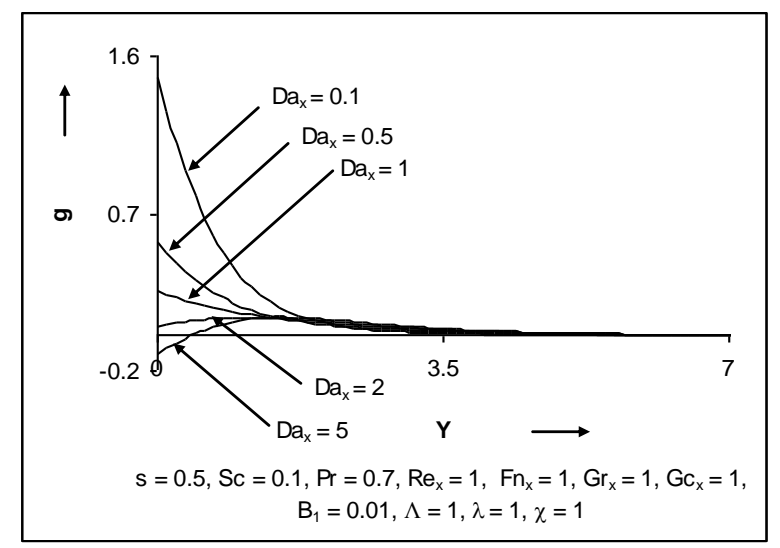

Fig 13 Microrotation distribution for different $\mathrm{Da}_{\mathrm{x}}$

The influence of the local porous media inertia parameter, $\mathrm{Fn}_{\mathrm{x}}$, on the flow regime is studied in Fig. 14 to 15 , for the reactive case. Velocity, (from Fig. 14) evidently falls drastically as $\mathrm{Fn}_{\mathrm{x}}$ increases. In particular, velocity near the stretching surface is sufficiently reduced and a flattening of the profiles occurs. In the momentum equation (5.9) the Forchheimer inertial drag is directly proportional to the $\mathrm{Fn}_{\mathrm{x}}$ number. Therefore for a fixed $\mathrm{Da}_{\mathrm{x}}=1$, large values of $\mathrm{Fs}_{\mathrm{x}}$ will strongly decelerate the flow regime, as justified by our computations.

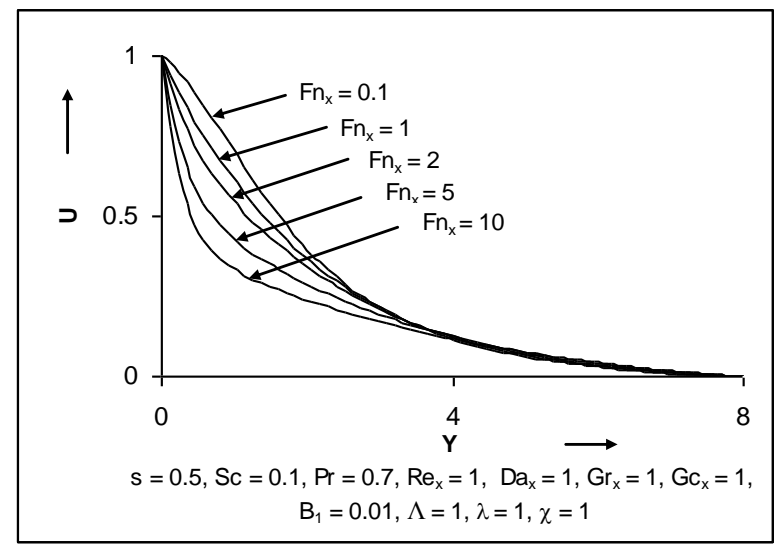

Fig 14 Velocity distribution for different $F n_{x}$

Micro-rotation function $\mathrm{g}$ as shown in Fig. 15 strongly increases in the near-wall region as $\mathrm{Fn}_{\mathrm{x}}$ increases Forchheimer drag therefore has a positive influence on angular velocity, but depresses translational velocities. 


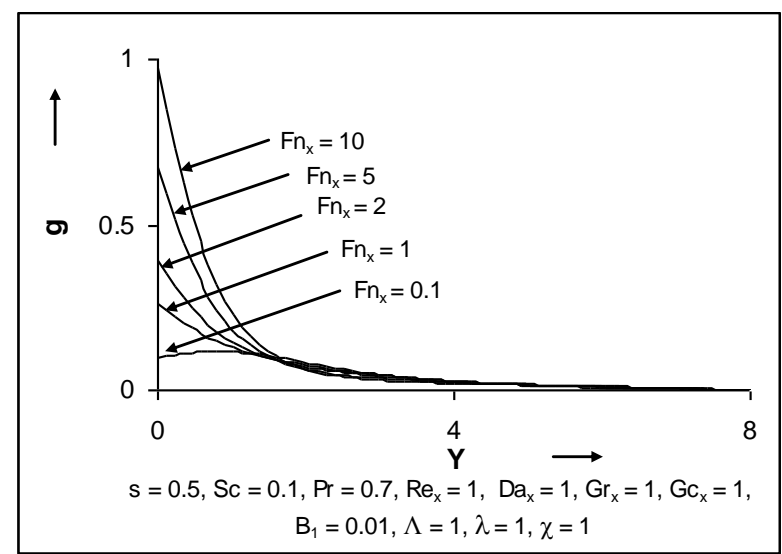

Fig 15 Microrotation distribution for different $\mathbf{F n}_{\mathbf{x}}$

The effect of Schmidt number (Sc) on the mass transfer function is illustrated for both the reactive flow case and the non-reactive flow case, in Figs. 16 and 17. Sc quantifies the relative effectiveness of momentum and species transfer by diffusion. Smaller Sc values can represent, for example hydrogen gas as the species diffusing $(\mathrm{Sc}=0.1$ to 0.2 ). $\mathrm{Sc}=1.0$ corresponds approximately to Carbon Dioxide diffusing in air, $\mathrm{Sc}=2.0$ implies Benzene diffusing in air, and higher values to petroleum derivatives diffusing in air (e.g. Ethylbenzene) as indicated by Gebhart et al [24]. Computations have been performed for $\operatorname{Pr}=0.7$, so that $\operatorname{Pr} \neq$ Sc, and physically this implies that the thermal and species diffusion regions are of different extents. As Sc increases, for the reactive flow case, Concentration strongly reduces, since larger values of Sc are equivalent to a reduction in the chemical molecular diffusivity i.e. less diffusion therefore takes place by mass transport. All profiles are seen to descend from a maximum concentration of 1 at $\mathrm{Y}=0$ (the wall) to zero. However, we observe a sharp decay in concentration profiles for high value of Sc, which becomes zero as early as $\mathrm{Y}=1$ approximately. For lower value of $\mathrm{Sc}$, a more gradual decay occurs to the free stream.

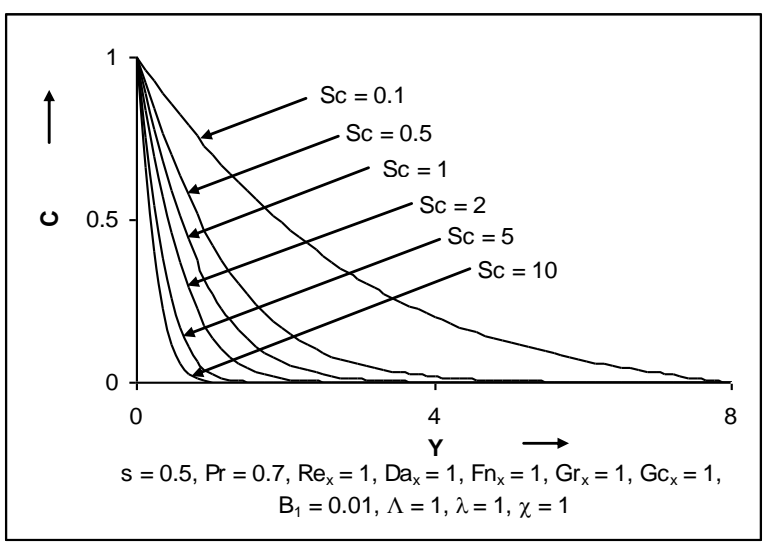

Fig. 16 Concentration distribution for different Sc

The influence of Sc on the concentration profiles for the non-reactive flow case is illustrated in Fig. 17. Although the trends are similar as for the reactive flow case, the profiles are less decreased with a rise in Sc, when chemical reaction is absent. For $\mathrm{Sc}=0.1$, there is almost a linear decay in the nonreactive case, whereas it is considerably parabolic for the reactive case, indicating lower values of concentration throughout the flow domain for the reactive case. Thus, it can be concluded that, in consistency with our earlier computations, chemical reaction decreases mass transfer markedly throughout the porous medium.

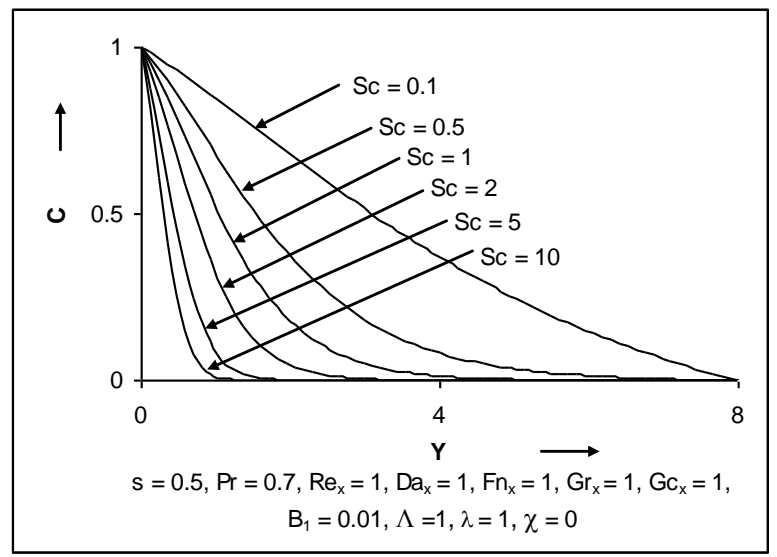

Fig. 17 Concentration distribution for different Sc (for $\chi=$ 0)

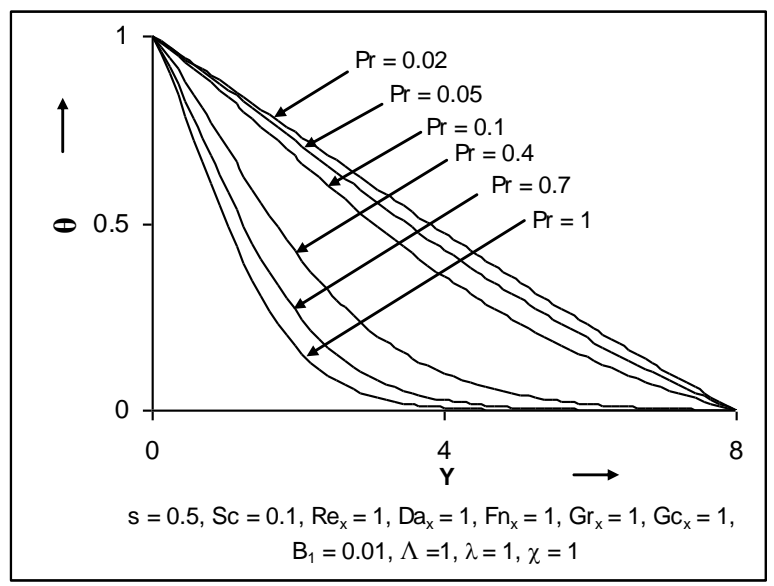

\section{Fig. 18 Temperature distribution for different Pr}

The influence of Prandtl number $\mathrm{Pr}$, on the temperature distribution is plotted in Fig. 18. Pr encapsulates the ratio of momentum diffusivity to thermal diffusivity. Larger Pr values imply a thinner thermal boundary layer thickness and more uniform temperature distributions across the boundary layer. For smaller values of Pr, fluids have higher thermal conductivy so that heat can diffuse away from the vertical surface faster than for higher Pr fluids (thicker boundary layers). Physically the lower values of $\operatorname{Pr}(\operatorname{Pr} \sim 0.02$, $0.05)$ correspond to liquid metals, $\operatorname{Pr}=0.7$ is accurate for air or hydrogen and $\operatorname{Pr}=1$ for water. The computations indicate that a rise in Pr substantially reduces the temperatures in the micropolar-fluid-saturated porous regime, a result consistent with other studies on coupled heat and mass transfer in porous media, see for example Kim [25]. In all cases, $\theta$ descends steadily to zero as $\mathrm{Y} \rightarrow \infty$, although the profile for maximum $\operatorname{Pr}(=1)$ is highly parabolic.

The influence of surface parameter $s$, on flow profile is indicated in Fig. 19. Micro-rotation is seen to increase substantially near the wall, as $\mathrm{s}$ increase. $\mathrm{s}=0$ implies that micro-rotation at the wall is prohibited explaining the zero value of micro-rotation for this case. As s increases, the microelements rotate with increasing intensity and this leads to the maximum angular velocity $\mathrm{g}$, at $\mathrm{s}=1.0$ at the 
wall. All profiles converge to a specific value of $\mathrm{Y}$ and since this location is far from the wall, the surface parameter, s, ceases to have any influence on the micro-rotation field here and beyond.

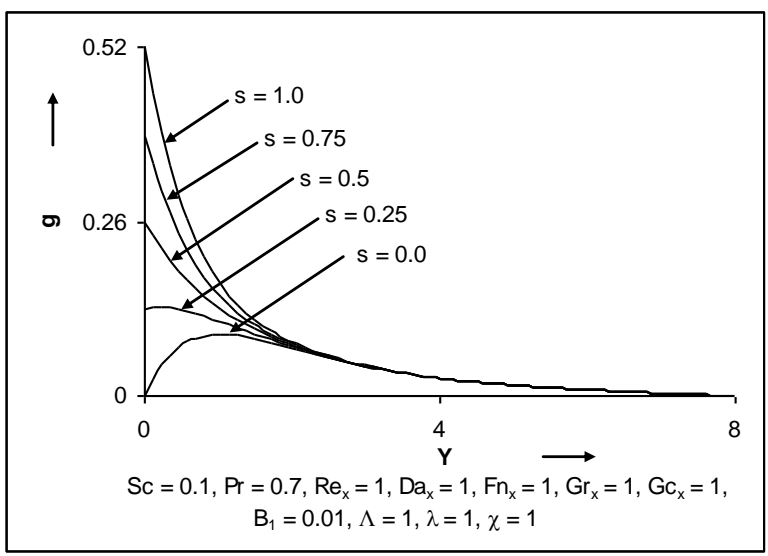

Fig. 19 Microrotation distribution for different $s$

A comparision of the results by finite element method and finite difference method has been given in Table 1. It is evident from the table 5.1, that the results obtained by the two techniques are in good agreement.

\begin{tabular}{|c|c|c|c|c|}
\hline \multicolumn{5}{|c}{ U } \\
\hline$Y$ & FEM & FDM & FEM & FDM \\
\hline 0 & 1 & 1 & 0.259095 & 0.259012 \\
\hline 0.8 & 0.673322 & 0.673285 & 0.148205 & 0.148164 \\
\hline 1.6 & 0.453918 & 0.453893 & 0.093566 & 0.093535 \\
\hline 2.4 & 0.297017 & 0.297001 & 0.061379 & 0.061357 \\
\hline 3.2 & 0.191239 & 0.191227 & 0.040084 & 0.040072 \\
\hline 4 & 0.121795 & 0.121791 & 0.026326 & 0.026319 \\
\hline 4.8 & 0.075524 & 0.075505 & 0.017486 & 0.017467 \\
\hline 5.6 & 0.043871 & 0.043843 & 0.011467 & 0.011436 \\
\hline 6.4 & 0.022071 & 0.022031 & 0.006937 & 0.006902 \\
\hline 7.2 & 0.007742 & 0.007699 & 0.003192 & 0.003145 \\
\hline 8 & 0 & 0 & 0 & 0 \\
\hline
\end{tabular}

Table 1. Comparison of FEM and FDM Computations $\left(s=0.5, S c=0.1, \operatorname{Pr}=0.7, \operatorname{Re}_{x}=1, D a_{x}=1.0, F n_{x}=1.0\right.$, $G r_{x}=1, G c_{x}=1, B_{1}=0.01, \Lambda=1, \lambda=1, \chi=1$

Table 2. contains the comparison of velocity $U$ and temperature $\theta$, as mentioned there, taking the linear and quadratic elements. It can be clearly seen that the results obtained using linear element matches to a good degree of accuracy, with those obtained by taking quadratic elements.

\begin{tabular}{|c|c|c|c|c|}
\hline \multirow{2}{*}{$Y$} & \multicolumn{2}{|c|}{$\mathrm{U}$} & \multicolumn{2}{c|}{$\theta$} \\
\cline { 2 - 5 } & Linear & Quadratic & Linear & Quadratic \\
\hline 0 & 1 & 1 & 1 & 1 \\
0.8 & 0.673322 & 0.673335 & 0.679604 & 0.679623 \\
1.6 & 0.453918 & 0.453931 & 0.369045 & 0.369057 \\
2.4 & 0.297017 & 0.297025 & 0.170441 & 0.170454 \\
3.2 & 0.191239 & 0.191247 & 0.070471 & 0.070486 \\
4 & 0.121795 & 0.121798 & 0.027049 & 0.027053 \\
4.8 & 0.075524 & 0.075532 & 0.009851 & 0.009861 \\
5.6 & 0.043871 & 0.043885 & 0.003423 & 0.003433 \\
6.4 & 0.022071 & 0.022088 & 0.001105 & 0.001117 \\
7.2 & 0.007742 & 0.007761 & 0.000286 & 0.000298 \\
8 & 0 & 0 & 0 & 0 \\
\hline
\end{tabular}

Table 2: Comparison of velocity function with linear as well as quadratic elements

$\left(\begin{array}{c}s=0.5, S c=0.1, \operatorname{Pr}=0.7, \operatorname{Re}_{x}=1, D a_{x}=1.0, F n_{x}=1.0, \\ G r_{x}=1, G c_{x}=1, B_{1}=0.01, \Lambda=1, \lambda=1, \chi=1\end{array}\right)$

The variation of skin friction and the heat transfer parameter with respect to $G r_{x}, G c_{x}, \chi$ and $D a_{x}$ has been given in Table $3 \mathrm{a}$ and $3 \mathrm{~b}$.

\begin{tabular}{|c|c|c|}
\hline \multicolumn{3}{|c|}{$\begin{array}{c}\mathrm{s}=0.5, \mathrm{Sc}=0.1, \operatorname{Pr}=0.7, \mathrm{Re}_{\mathrm{x}}=1, \mathrm{Da}_{\mathrm{x}}=1, \mathrm{Fn}_{\mathrm{x}}=1, \mathrm{Gc}_{\mathrm{x}}= \\
1, \mathrm{~B}_{1}=0.01, \Lambda=1, \lambda=1, \chi=1\end{array}$} \\
\hline$G r_{x}$ & $f^{\prime \prime}(0)$ & $-\theta^{\prime}(0)$ \\
\hline 1 & -0.51819 & 0.33434 \\
\hline 2 & -0.151025 & 0.35892 \\
\hline 3 & 0.193374 & 0.37878 \\
\hline 5 & 0.83536 & 0.41037 \\
\hline 10 & 2.270969 & 0.46629 \\
\hline \multicolumn{3}{|c|}{$\begin{array}{c}\mathrm{s}=0.5, \mathrm{Sc}=0.1, \operatorname{Pr}=0.7, \mathrm{Re}_{\mathrm{x}}=1, \mathrm{Da}_{\mathrm{x}}=1, \mathrm{Fn}_{\mathrm{x}}=1, \\
\mathrm{Gr}_{\mathrm{x}}=1, \mathrm{~B}_{1}=0.01, \quad \chi=1, \Lambda=1, \quad \lambda=1\end{array}$} \\
\hline$G c_{x}$ & $f^{\prime \prime}(0)$ & $-\theta^{\prime}(0)$ \\
\hline 0.1 & -0.906182 & 0.28986 \\
\hline 1 & -0.51819 & 0.33429 \\
\hline 3 & 0.280086 & 0.39946 \\
\hline 5 & 1.01595 & 0.44396 \\
\hline 7 & 1.70756 & 0.47859 \\
\hline
\end{tabular}

Table 3a. Table for skin friction $\left\{f^{\prime \prime}(0)\right\}$ and the rate of heat transfer $\left\{-\theta^{\prime}(0)\right\}$ with different value of Grashof number $G r_{x}$ and Buoyancy parameter $G c_{x}$ 


\begin{tabular}{|c|c|c|}
\hline \multicolumn{3}{|c|}{$\mathrm{s}=0.5, \mathrm{Sc}=0.1, \mathrm{Pr}=0.7, \mathrm{Re}_{\mathrm{x}}=1, \mathrm{Fn}_{\mathrm{x}}=1, \mathrm{Gr}_{\mathrm{x}}=1}$, \\
$\mathrm{Gc}_{\mathrm{x}}=1, \mathrm{~B}_{1}=0.01, \chi=1, \Lambda=1$, & $\lambda=1$ \\
\hline$D a_{x}$ & $f^{\prime \prime}(0)$ & $-\theta^{\prime}(0)$ \\
\hline 0.1 & -2.96021 & 0.18792 \\
0.5 & -1.07287 & 0.29256 \\
1 & -0.51819 & 0.33429 \\
2 & 0.118063 & 0.36480 \\
5 & 0.200286 & 0.38829 \\
\hline $\mathrm{s}=0.5, \mathrm{Sc}=0.1, \mathrm{Pr}=0.7, \mathrm{Re}_{\mathrm{x}}=1, \mathrm{Da}=1, \mathrm{Fn}_{\mathrm{x}}=1$, \\
$\mathrm{Gr}_{\mathrm{x}}=1, \mathrm{Gc}_{\mathrm{x}}=1, \mathrm{~B}_{1}=0.01, \Lambda=1$, & $\lambda=1$ \\
\hline$\chi$ & $f^{\prime \prime}(0)$ & $-\theta^{\prime}(0)$ \\
\hline 0 & -0.468036 & 0.347138 \\
1 & -0.518191 & 0.334293 \\
5 & -0.599058 & 0.314798 \\
10 & -0.644068 & 0.306022 \\
20 & -0.692132 & 0.298698 \\
\hline
\end{tabular}

Table 3b. Table for skin friction $\left\{f^{\prime \prime}(0)\right\}$ and the rate of heat transfer $\left\{-\theta^{\prime}(0)\right\}$ with different value of Chemical

\section{reaction number $\chi$ and Darcy number $D a_{r}$.}

It is observed that both the coefficient of skin friction and the rate of heat transfer increases with the increase in $G r_{x}, G c_{x}$ and $D a_{x}$. However an increase in chemical reaction parameter leads to a decrease in coefficient of skin friction as well as rate of heat transfer. This implies that the parameters $G r_{x}, G c_{x,} \chi$ and $D a_{x}$ are effective not only in controlling skin friction, but also rate of heat transfer.

\section{CONCLUSIONS}

The numerical simulations indicate that:

(a) Translational velocity decreases, temperature increases, micro-rotation increases (in the near-field and intermediate range from the wall) and mass transfer function decreases with a rise in chemical reaction parameter $(\chi)$.

(b) Increasing thermal Grashof number $\mathrm{Gr}_{\mathrm{x}}$, increases the translational velocity, decreases temperature function values and decreases micro-rotation, the latter in the regime near the wall.

(c) Increasing species Grashof number $\mathrm{Gc}_{\mathrm{x}}$, increases translational velocity, decreases temperature, decreases mass transfer function and lowers the microrotation at the wall.

(d) Increasing local Darcy number $\mathrm{Da}_{\mathrm{x}}$, increases translational velocities but reduces temperature and micro-rotation, in the latter case, again the depression is maximized at the stretching surface (wall).

(e) Increasing local Forchheimer number $\mathrm{Fn}_{\mathrm{x}}$, reduces translational velocities, but boosts the micro-rotation, in the latter case especially at the wall and near the wall. (f) Increasing Schmidt number reduces mass transfer function both in the reactive and non-reactive flow cases, although mass transfer function values are always higher for any Sc value in the non-reactive case $(\chi=0)$.

(g) Increasing Prandtl number substantially reduces temperature function $(\theta)$.

(h) Increasing the surface parameter substantially increases micro-rotation $\mathrm{g}$, particularly at and near the wall.

(i) An increase in $G r_{x}, G c_{x}$ and $D a_{x}$ lead to an increase in coefficient of skin friction and the rate of heat transfer.

(j) Coefficient of skin friction and rate of heat transfer decreases with the increase in chemical reaction parameter.

\section{REFERENCES}

[1] Bertolazzi E., A finite volume scheme for twodimensional chemically-reactive hypersonic flow, Int. J. Num. Meth. Heat Fluid Flow, 8 (8) (1998) 888-933.

[2] Keller J.O. and Daily J.W., The effects of highly exothermic chemical reaction on a two-dimensional mixing layer, AIAA J., 23 (1985)1937-1945.

[3] Kelemen P., Dick P. and Quick J., Production of harzburgite by pervasive melt rock-reaction in the upper mantle, Nature, 358 (1992) 635-641.

[4] Zeiser T., Lammers P., Klemm E., Li Y.W., Bernsdorf J. and Brenner G., CFD Calculation of flow, dispersion and reaction in a catalyst filled tube by lattice Boltzmann method, Chem. Eng. Sci., 56 (4) (2001) 1697-1704.

[5] Levenspiel O., Chemical Reaction Engineering, John Wiley, New York, $3^{\text {rd }}$ edition (1999).

[6] Acrivos A., On laminar boundary layer flows with a rapid homogenous chemical reaction, Chem. Eng. Sci. 13 (1960) 57.

[7] Takhar H.S. and Soundalgekar V.M., On the diffusion of a chemically reactive species in a laminar boundary layer flow past a porous plate. "L" Aerotechnica Missili E. Spazio. 58 (1980) 89-92.

[8] Merkin J.H. and Chaudhary M.A., Free convection boundary layers on vertical surfaces driven by an exothermic reaction, Q. J. Mechanics Applied Math. 47 (1994) 405-428.

[9] Shateyi S., Sibanda P. and Motsa S.S., An asymptotic analysis of convection in boundary layer flow in the presence of a chemical reaction, Archives of Mechanics, 57 (1) (2005) 24-41.

[10] Pop I., Soundalgekar V.M. and Takhar H.S., Dispersion of a soluble matter in a porous medium channel with homogenous and heterogenous chemical reaction. Revue Romanie. Mecanique Appliquee 28 (1983) 127-132.

[11] Aharonov E., Spiegelman M. and Kelemen P., Threedimensional flow and reaction in porous media: implications for the earth's mantle and sedimentary basins, J. Geophys. Res. 102 (1997) 14821-14834.

[12] Fogler H.S. and Fredd C., The influence of transport and reaction on wormhole formation in porous media, A I Chem E J. 44 (1998) 1933. 
[13] Sakiadis B.C., Boundary layer behaviour on continuous solid surface II: The boundary layer on a continuous flat surface, A I Chem E J. 7 (1961b) 221-225.

[14] Vlegger J., Laminar boundary layer behaviour on continuous accelerating surfaces, Chem. Eng. Sci. 32 (1977) 1517-1528.

[15] Takhar H.S., Chamkha A.J. and Nath G., Flow and mass transfer on a stretching sheet with a magnetic field and chemically reactive species, Int. J. Engineering. Science 38 (2000) 1303-1314.

[16] Acharya M., Singh L.P. and Dash G.C., Heat and mass transfer over an accelerating surface with heat source in the presence of suction/blowing, Int. J. Engineering Science 37 (1999) 189-201.

[17] Eringen A.C., Theory of Micropolar Fluids, J. Mathematics Mechanics 16 (1966) 1-18.

[18] Eringen A.C., Theory of Thermomicrofluids, Mathematical analysis and Applications Journal 38 (1972) 480-496.

[19] Hassanien I.A. and Gorla R.S.R., Mixed convection boundary layer flow of a micropolar fluid near a stagnation point on a horizontal cylinder, Int. J. Engng Sci., 28 (1990) 153-161.

[20] Agarwal R.S., Bhargava R. and Balaji A.V.S., Finite element solution of flow and heat transfer of a micropolar fluid over a stretching sheet, Int. J. Engineering Science, 27 (1989) 1421-1440.

[21] Bhargava R., Takhar H.S., Agarwal R.S. and Balaji A.V.S., Finite element solution of micropolar fluid flow and heat transfer between two porous discs, Int. J. Engineering Science 38 (2000) 1907-1922.

[22] Crane L.J., Flow past a stretching plate, J. Applied Mathematics and Physics, ZAMP 21 (1970) 645-657.

[23] Afify A.A., MHD free convective flow and mass transfer over a stretching sheet with chemical reaction, Heat and Mass Transfer 40 (2004) 495-500.
[24] Gebhart B., Jaluria Y., Mahajan R.L., Sammakia B., Buoyancy-induced flows and transport, Hemisphere USA (1998).

[25] Kim Y.J., Heat and mass transfer in MHD micropolar flow over a vertical moving porous plate in a porous medium, Transp. Porous Media J. 56 (2004) 17-37.

[26] Beg O. A., Bhargava R. , Rawat S., Takhar H.S., Beg T. A. "A Study of Steady Buoyancy- Driven Dissipative Micropolar Free Convective Heat and Mass Transfer in a Darcian Porous Regime with Chemical Reaction ., Nonlinear Analysis: Modeling and Control, , 12, 2, 157180 (2007)

[27] Bég O. A., Bhargava R. , Rawat S. , Kalim Halim and Takhar H. S. "Computational modeling of biomegnatics micropolar blood flow and heat transfer in a two dimensional non-darcian porous channel. Meccanica 43: 391-410 (2008)

[28] S. Rawat \& R. Bhargava ." Finite element study of natural convection heat and mass transfer in a micropolar fluid-saturated porous regime with soret/dufour effects ., Int. J. of Appl. Math and Mech. 5, 2, 58-71. (2009)

[29] S. Rawat, R. Bhargava and O. Anwar Bég., "Hydromagnetic micropolar free convection heat and mass transfer in a darcy-forchheimer porous medium with thermophysical effects: finite element solutions ., Int. J. of Appl. Math and Mech, 6, 13, $72-93$ (2010)

[30] H. Usman M. M. Hamza B.Y Isah ., Unsteady MHD Micropolar Flow and Mass Transfer Past a Vertical Permeable Plate with Variable Suction., International Journal of Computer Applications 36(4), 2011

[31] H. Usman M. M. Hamza M.O Ibrahim., RadiationConvection Flow in Porous Medium with Chemical Reaction., International Journal of Computer Applications 36(2), 2011 